\title{
Passages à l'agriculture biologique. Une diversité de processus
}

Transitions to organic farming: a diversity of processes

\section{Françoise Alavoine-Mornas et Sophie Madelrieux}

\section{(2) OpenEdition}

\section{Édition électronique}

URL : http://journals.openedition.org/economierurale/4235

DOI : 10.4000/economierurale.4235

ISSN : 2105-2581

\section{Éditeur}

Société Française d'Économie Rurale (SFER)

\section{Édition imprimée}

Date de publication : 20 janvier 2014

Pagination : 65-79

ISSN : 0013-0559

\section{Référence électronique}

Françoise Alavoine-Mornas et Sophie Madelrieux, « Passages à l'agriculture biologique. Une diversité de processus », Économie rurale [En ligne], 339-340 I janvier-mars 2014, mis en ligne le 20 janvier 2016, consulté le 19 avril 2019. URL : http://journals.openedition.org/economierurale/4235 ; DOI : 10.4000/ economierurale.4235 


\section{Passages à l'agriculture biologique Une diversité de processus}

Françoise ALAVOINE-MORNAS, Sophie MADELRIEUX • Irstea, UR Développement des Territoires Montagnards, Saint-Martin-d'Hères

francoise.alavoine-mornas@irstea.fr

Tandis que la consommation de produits bio augmente, l'agriculture biologique présente des dynamiques contrastées selon les régions et les productions. Elle est également l'objet de controverses quant à sa conventionalisation (évolution vers une version modifiée de l'agriculture conventionnelle). Les auteures se sont interrogées sur la façon dont des exploitants en viennent à I'agriculture biologique et ses formes d'exercice. À partir de l'étude de différents cas de passage à I'AB en Rhône-Alpes, les auteures analysent la place des certifications $A B$ dans les cheminements des exploitants et des exploitations. Les résultats montrent une diversité de processus, loin des dualismes présents dans la littérature : AB/conventionnel, bio-éthique/bio-pragmatique...

MOTS-CLÉS : agriculture biologique, exploitation agricole, certification, changement, trajectoires

\section{Transitions to organic farming: a diversity of processes}

Facing an increasing supply of organic produces, organic farming shows various dynamics depending on productions and regions. There is also a controversy about the possible evolution of organic farming to a modified version of conventional agriculture. Therefore we try to understand the ways farmers come to organic farming, but also the forms it takes. Basing on a case study in Rhône-Alpes, we analyse the different places occupied by the certification in farmers and farms pathways. Our results show the diversity of processes, far from the dualisms so often present in the literature: organic/conventionally, bio-ethic/bio-pragmatic... (JEL: Q01, Q12).

KEYWORDS: organic agriculture, farm, certification, change, path

\section{Quels développements de l'agriculture biologique au niveau des exploitations agricoles?}

L'Agriculture biologique (AB), reposant sur une réglementation européenne basée sur une obligation de moyens (incluant principalement l'interdiction des produits de synthèse), est considérée comme un modèle d'agriculture durable (Rigby et Caceres, 2001). En tant que telle, elle est soutenue par les politiques publiques. La stagnation des conversions d'exploitations agricoles à l' AB en France, de 2002 à 2007, a été suivie, depuis 2008, par une reprise contrastée selon les régions et les productions (Agence Bio, 2012). Parallèlement, la consommation de produits issus de l'AB augmente, et l'offre ne parvient pas à répondre à la demande. En 2010, $35 \%$ de la valeur des produits bio consommés en France proviennent d'un autre pays, dont le tiers pour lequel la France a une vocation naturelle, mais manque de volumes (Agence Bio, 2012). Ces évolutions interrogent sur les freins au développement de l'agriculture biologique dans les exploitations agricoles et ses leviers. Certains auteurs pointent dans le même temps une tendance à la « conventionalisation »de l'AB (Guthman, 2004), ce qui pose la question des formes d'exercice de l'agriculture biologique à encourager. Cette conventionalisation reposerait sur une évolution de l'AB vers une version modifiée de l'agriculture conventionnelle (économies d'échelle, substitution de ressources, recours croissant à des intrants...), avec 
une centralité des objectifs économiques, qui, si elle se généralisait, pourrait compromettre la contribution de l'AB à un développement durable (Darnhofer et al., 2010). Au niveau des exploitations, cela se traduit par un éloignement par rapport aux principes fondateurs du bio (principes de santé, d'écologie, de justice, de précaution) (IFOAM ${ }^{1}$, 2005).

Nous nous interrogeons dans cet article sur la manière dont les exploitants en viennent à une certification $\mathrm{AB}$, avec quelles formes d'exercice de l'agriculture biologique (les pratiques au niveau de l'exploitation et le positionnement des exploitants par rapport aux principes fondateurs du bio), en nous appuyant sur une étude de différents cas de passage à l'AB en RhôneAlpes. Dans la suite du texte, AB fera référence à la certification européenne ; bio aux principes fondateurs de l'IFOAM, qui dépassent d'ailleurs le seul domaine agricole pour toucher tous les domaines de la vie, et conventionnel sera utilisé pour signifier non $\mathrm{AB}$ et non bio.

\section{Du brouillage et des dualismes aux processus de passage à l'AB}

L'examen de la littérature confronté à nos observations de terrain suggère que la compréhension des passages à l'agriculture biologique est rendue difficile par l'existence de trois brouillages. Les exploitants, quand ils certifient leur exploitation en $A B$, peuvent s'engager dans différentes pratiques de l'agriculture biologique, ce qui constitue un premier niveau de brouillage. Certains se conforment au seul respect de la réglementation, avec même parfois mise en place de pratiques non durables, mais pour autant non interdites par le cahier des charges de l'AB (Padel et al., 2007). D'autres mettent en place des

1. IFOAM : International Federation of Organic Agriculture Movements. pratiques plus exigeantes que le seul cahier des charges $\mathrm{AB}$, dans le sens des principes fondateurs du bio. Certains auteurs les nomment beyond organic (Nelson et al., 2010). Enfin, d'autres agriculteurs optent pour une certification partielle et font coexister sur leur exploitation agriculture biologique et agriculture conventionnelle. Ces certifications partielles concernent en 2011 le quart des exploitations avec une certification $A B$ et plus du tiers des exploitants nouvellement engagés en 2011 (Agence Bio, 2012). Or la littérature se limite souvent à évoquer le dualisme $\mathrm{AB}$ versus conventionnel et ne prend notamment pas en considération ces cas de certification partielle (Guthman, 2000).

Une deuxième confusion provient d'un manque de distinction entre exploitations et individus, sans doute du fait du cloisonnement des approches entre sciences sociales et sciences techniques, comme le soulignent Lamine et Bellon (2009) dans leur revue de la littérature concernant les conversions à l'agriculture biologique. Mais là encore les dualismes du type bio-engagé versus bio-pragmatique (Darnhofer, et al., 2005 ; Fairweather J., 1999) ne rendent pas compte d'une réalité souvent plus nuancée : exploitations certifiées $\mathrm{AB}$ dont les exploitants ne sont pas motivés à titre personnel par les principes fondateurs du bio, exploitants dans un mode de vie bio sans que leur exploitation ne soit certifiée, ou encore exploitants jouant sur plusieurs registres - bio, $\mathrm{AB}$ ou conventionnel - pour différents motifs restant à décrypter. Des convergences et des tensions se nouent ainsi entre bio, $\mathrm{AB}$ et conventionnel au niveau des exploitations comme des individus, brouillant les cartes et témoignant de processus complexes.

Un troisième brouillage provient des temporalités considérées. La littérature minimise en effet souvent l'importance du temps. Au contraire, Lamine et Bellon (2009) considèrent la conversion à l'AB 
comme un processus plus long que la durée légale de conversion, mettant en jeu des recompositions diverses (les terres, les troupeaux, les productions, les individus, les réseaux..., et leurs relations). Ces auteurs considèrent ainsi que c'est la combinaison d'éléments déclencheurs, de motivations, de facteurs favorisants, qui aboutissent à la décision de conversion. Des approches par les récits de vie témoignent de tensions identitaires qui peuvent se nouer au niveau des individus, pris entre exploitation, famille, regard des autres, en amont de la certification, et montrent comment des évènements biographiques peuvent aider à franchir le pas de l'AB (Van Dam, 2005). De nombreux travaux ont étudié les motivations à la conversion, mais peu d'auteurs ont choisi de suivre les processus de passage à l' $\mathrm{AB}$ au niveau des exploitations et des exploitants, et d'analyser les changements dans les conceptions et les pratiques, les évolutions dans le temps (Koesling et al., 2012 ; Lamine 2011), même si de telles approches étaient au cour des travaux pionniers sur l'agriculture biologique (Harris et al., 1979 ; Barrès et al., 1985 ; Le Pape et Remy, 1988). Lamine et al. (2009) mettent ainsi en évidence des conversions directes au sens où la certification concerne d'emblée la totalité de l'exploitation, et des passages à l' $\mathrm{AB}$ indirects et progressifs, au sens où la première certification concerne une partie seulement de l'exploitation tandis que d'autres parties sont certifiées par la suite. Adopter une telle approche par les processus, c'est-à-dire à la fois dynamique et contextuelle (Pettigrew, 1990) signifie passer d'une approche causale (le pourquoi) à une approche séquentielle (le comment). Cela permet également un examen détaillé des circonstances entourant la décision de conversion, alors que les contextes des changements dans les exploitations ont été insuffisamment pris en compte, notamment la combinaison d'éléments internes et externes à l'exploitation (Evans, 2009).
Pour contribuer à l'identification des chemins possibles vers et dans l'agriculture biologique, nous proposons d'examiner dans cet article les processus de passage à la certification AB. Nous examinons plus particulièrement la place de la certification $\mathrm{AB}$ dans les cheminements des exploitants et des exploitations. Pour cela, nous décomposons le processus de passage à l'AB en nous concentrant tout d'abord sur la première certification $A B$, les circonstances qui y ont conduit et sa nature, partielle ou totale. Puis nous nous focalisons sur la suite du processus, dans les cas où la première certification a été partielle, et sur la place de ces certifications partielles dans les cheminements. Nous formulons l'hypothèse que selon le processus de passage à l'AB, l'engagement dans le bio et l'AB n'est pas le même.

\section{Étude de différents cas de passage à l'agriculture biologique}

Dans le cadre du projet, L'Agriculture Biologique, un prototype d'agriculture pour un développement durable, coordonné par l'Isara-Lyon, nous avons mené une étude de différents cas de passage à l'AB. Le choix des exploitations enquêtées a été fait pour couvrir une diversité de dates de certification $A B$, de systèmes de production, de collectifs de travail, de dynamiques territoriales par rapport à l'AB. Les buts étaient d'éviter que notre analyse ne soit biaisée par des effets sectoriels ou territoriaux trop marqués, mais aussi d'accroître les sources possibles de variation des processus en jeu.

L'objectif des entretiens était de resituer le passage à l'AB dans l'histoire des individus, de la famille, et dans celle de l'exploitation. L'entretien, de type récit de vie (Bertaux, 1997), a été réalisé avec le chef d'exploitation et, lorsqu'il y avait plusieurs associés, avec un de ceux qui a été moteur dans le passage à l' $\mathrm{AB}$, tout en cherchant à saisir le positionnement des différents 
Passages à l'agriculture biologique

Tableau 1.Présentation des exploitations enquêtées en 2010 et de leurs certifications $A B$

\begin{tabular}{|c|c|c|c|}
\hline $\begin{array}{l}\text { Cas } \\
\text { (dptt) }\end{array}$ & $\begin{array}{l}\text { Collectif de travail } \\
\text { sur l'exploitation }\end{array}$ & $\begin{array}{c}\text { Combinaison d'activités (dont } \\
\text { système } \\
\text { de production) }\end{array}$ & $\begin{array}{l}\text { Certifications AB de l'exploitation (EA) } \\
\text { (date de la } 1^{\text {re }} \text { certification) }\end{array}$ \\
\hline \multicolumn{4}{|c|}{ L'officialisation } \\
\hline$B(26)$ & Couple & Ovin lait + fabrication et vente & Certification directe et totale (2000) \\
\hline$C(26)$ & GAEC parents-fils & Ovin lait + fabrication et vente & Certification directe et totale (2009) \\
\hline$F(26)$ & $\begin{array}{l}\text { Exploitant + aide } \\
\text { famille }\end{array}$ & $\begin{array}{l}\text { Ovin viande + transformation } \\
\text { et vente }\end{array}$ & $\begin{array}{l}\text { Certification directe et totale (1995) (puis } \\
\text { sortie partielle de l'AB) }\end{array}$ \\
\hline$K(26)$ & Couple & Bovin viande & Certification directe et totale (2008) \\
\hline $0(26)$ & Couple + fille & $\begin{array}{l}\text { Arboriculture + transforma- } \\
\text { tion et vente + cultures }\end{array}$ & $\begin{array}{l}\text { Certifications progressives (résolution de diffi- } \\
\text { cultés) jusqu'à la totalité de l'EA (1982) }\end{array}$ \\
\hline \multicolumn{4}{|c|}{ L'imprévu } \\
\hline$A(38)$ & Couple & Bovin lait & $\begin{array}{l}\text { Certification directe et totale (2000) (puis } \\
\text { sortie totale de l'AB) }\end{array}$ \\
\hline G (26) & $\begin{array}{l}\text { Exploitant + aide } \\
\text { famille }\end{array}$ & $\begin{array}{l}\text { Ovin viande + lavande } \\
\text { Déneigement l'hiver }\end{array}$ & $\begin{array}{l}\text { Certifications progressives (précaution) } \\
\text { jusqu'à la totalité de l'EA (1996) }\end{array}$ \\
\hline$M(26)$ & $\begin{array}{l}\text { Couple + fille + } \\
\text { salariés }\end{array}$ & Arboriculture + cultures & $\begin{array}{l}\text { Certifications progressives (précaution), mais } \\
\text { qui restent partielles (difficultés) (1998) }\end{array}$ \\
\hline \multicolumn{4}{|c|}{ L'opportunité } \\
\hline$H(26)$ & $\begin{array}{l}\text { Exploitant + } \\
\text { salariés }\end{array}$ & $\begin{array}{l}\text { Ovin viande + transformation } \\
\text { et vente }+ \text { cultures }\end{array}$ & Certification directe et totale (2001) \\
\hline$P(26)$ & $\begin{array}{l}2 \text { frères (EARL) + } \\
\text { salariés }\end{array}$ & Arboriculture + cultures & $\begin{array}{l}\text { Une certification partielle (rationalisation) } \\
(2008)\end{array}$ \\
\hline \multicolumn{4}{|c|}{ Résolution de tensions au niveau individuel } \\
\hline$D(26)$ & $\begin{array}{l}\text { Exploitant + aide } \\
\text { famille }\end{array}$ & \begin{tabular}{|l|} 
Ovin et bovin viande + \\
cultures \\
Ramassage scolaire + location
\end{tabular} & Certification directe et totale (2010) \\
\hline$E(26)$ & $\begin{array}{l}\text { Exploitant + } \\
\text { salariés }\end{array}$ & $\begin{array}{l}\text { Ovin viande + poules pon- } \\
\text { deuses + cultures }\end{array}$ & $\begin{array}{l}\text { Certifications progressives (précaution) } \\
\text { jusqu'à la totalité de l'EA (1996) }\end{array}$ \\
\hline$I(26)$ & $\begin{array}{l}\text { GAEC à } 5 \text { associés } \\
\text { + salariés }\end{array}$ & Poly-élevages + cultures & $\begin{array}{l}\text { Certifications progressives (négociation), mais } \\
\text { qui restent partielles (rationalisation) (1997) }\end{array}$ \\
\hline$J(26)$ & Couple + aide fils & $\begin{array}{l}\text { Ovin viande + cultures } \\
\text { Gîte + abbaye (stages, } \\
\text { concerts...) }\end{array}$ & $\begin{array}{l}\text { Certifications progressives (précaution), mais } \\
\text { qui restent partielles (rationalisation) (1986) }\end{array}$ \\
\hline$L(26)$ & $\begin{array}{l}\text { Exploitant + } \\
\text { salariés }\end{array}$ & Arboriculture + cultures & $\begin{array}{l}\text { Une certification partielle (hypothèse } \\
\text { d'attente d'opportunité) (2009) }\end{array}$ \\
\hline$N(26)$ & 2 frères (EARL) & $\begin{array}{l}\text { Arboriculture + cultures }+ \\
\text { entreprise de travaux agricoles }\end{array}$ & $\begin{array}{l}\text { Une certification partielle (hypothèse de } \\
\text { négociation) (2009) }\end{array}$ \\
\hline $\mathrm{Q}(42)$ & Couple & Bovin lait & Certification directe et totale (2009) \\
\hline $\mathrm{R}(42)$ & $\begin{array}{l}\text { GAEC familial à } 3 \\
\text { associés + salarié }\end{array}$ & $\begin{array}{l}\text { Bovin lait + cultures + ferme } \\
\text { pédagogique }\end{array}$ & Certification directe et totale (2008) \\
\hline
\end{tabular}

Note : dptt : département

Source : les auteures.

membres de la famille et du collectif de travail par rapport au bio, et leur influence sur le passage à l'AB. Dans chaque entretien, d'une durée de 1,5 à 2,5 heures, nous avons cherché à préciser les antécédents, les éléments déclencheurs ou les circonstances de cette certification, les éléments du choix de l'AB, les facteurs l'ayant favorisé, les transitions ou les ruptures entre modes de production (Sylvander et al., 2006 ; 
RECHERCHES

Françoise ALAVOINE-MORNAS, Sophie MADELRIEUX

Lamine et Bellon, 2009). Chaque entretien a donné lieu à la production de deux types de documents : i) une monographie de type narratif visant à décrire les processus de passage à l'AB (quand, pourquoi, comment, avec quelles implications) ; ii) une représentation graphique des changements $\mathrm{du}$ système famille-exploitation et des éléments de contexte internes et externes à l'exploitation ayant conduit à ces changements. À partir de ces documents, nous avons examiné le cheminement des individus et des exploitations par rapport à l'AB, mais aussi par rapport aux principes du bio. Nous avons considéré qu'un individu (une famille) cheminait dans le bio quand il cherchait à mettre en accord ses pratiques de production, de consommation, de soins (alimentation, santé, consommation d'énergie) avec les principes fondateurs du bio.

La comparaison des différents cas nous a permis, de manière itérative, d'identifier les concepts permettant de rendre compte de l'ensemble des processus à l'œuvre (Pettigrew, 1990). Les discussions entre les chercheurs ont permis de tester et d'affiner notre représentation des passages à l'AB, d'identifier les places de la certification $\mathrm{AB}$ dans les cheminements des exploitants et des exploitations, et de comprendre sa signification.

Nous avons réalisé des entretiens auprès de 18 exploitants. Le tableau 1 récapitule les cas étudiés à partir de leur localisation, des collectifs de travail et des combinaisons d'activités (dont le système de production). La quatrième colonne présente les éléments de qualification des circonstances et de la nature de la première certification $\mathrm{AB}$, et des certifications qui ont suivi lorsque la première était partielle. Nous précisons entre parenthèses la signification de ces certifications partielles, soit de leur succession dans le cas de certifications progressives, soit des raisons pour lesquelles, au moment de l'enquête, une partie seulement de l'exploitation était certifiée (ce qui sera détaillé dans la suite).

\section{Places et significations des certifications $A B$ dans les processus de passage}

\section{Place de la première certification $A B$ dans les cheminements}

Circonstances de la première certification $A B$

Les passages à l'agriculture biologique sont constitués d'un cheminement des exploitants, des activités et des pratiques sur l'exploitation, par rapport aux fondements du bio, et à la certification $\mathrm{AB}$ d'une (ou des) activité(s) de l'exploitation. La place qu'occupe une première certification $\mathrm{AB}$, qu'elle concerne toute l'exploitation ou une partie de celle-ci, semble renvoyer dans les cas rencontrés à quatre types de situation, que nous avons intitulés « officialisation », « imprévu », « opportunité », « résolution de tensions ». Ces types se réfèrent aux circonstances de la certification $\mathrm{AB}$, mais également aux interactions entre certification et cheminements des exploitants et des exploitations. Les cheminements des familles viennent compléter la caractérisation des types.

\section{- L'officialisation (5 cas)}

Dans ces exploitations, pratiquant toutes la transformation et la vente directe, les exploitants sont dans un cheminement ancien par rapport au bio, avec une philosophie de vie où les pratiques sur l'exploitation et celles de la famille sont en cohérence. Dans les exploitations d'élevage, la certification est assez récente et concerne la totalité de l'exploitation (comme dans le cas de B, encadré 1). Dans l'exploitation d'arboriculture fruitière, la certification, plus ancienne, était partielle au début. Dans tous les cas, elle est liée à la demande ou à des interrogations des clients, face auxquelles les exploitants ont ressenti le besoin de clarifier leur position. La certification ne s'accompagne pas d'un changement de mode de production ou de façon de concevoir leur activité, mais elle officialise une dynamique ancienne. 
La certification $\mathrm{AB}$ officialise un cheminement déjà ancien dans le bio des individus, des familles, des activités et des pratiques sur l'exploitation.

\section{Encadré 1. Cas de B}

Dans cette exploitation individuelle où le couple s'occupe de 120 brebis laitières, 50 ha de prairies, transforme et vend les fromages, l'engagement dans le bio comme mode de vie est ancien. Ils s'installent « hors du cadre familial » en 1987. Ils se forment à l'homéopathie, font des stages en fromagerie bio et sur l'utilisation de levain naturel, ils travaillent sur l'immunité des animaux par une «préservation de la biodiversité génétique », ils limitent les marchés et déplacements longs, en cohérence avec leurs pratiques familiales. Pour eux, l'agriculture biologique c'est « respecter les lois naturelles par rapport à la vie du sol et des animaux ». En 2000, ils certifient leur exploitation directement et sur la totalité, car les clients leur demandaient de plus en plus souvent s'ils étaient en $\mathrm{AB}$, et outre un discours sur leurs pratiques, le label permettait de donner une forme de garantie à ces clients.

\section{- L'imprévu (3 cas)}

Ce type rassemble des exploitations pour lesquelles la certification a pour origine un évènement inattendu, brutal, dans la famille (décès) ou l'exploitation (départ inattendu d'un associé comme dans le cas de $\mathrm{G}$, encadré 2, ou maladie décimant une production), qui amène l'exploitant à revoir la combinaison des activités. Le choix de l'AB est lié soit à une rencontre décisive, soit à une opportunité (opportunité de marché ou aide financière type Contrat territorial d'exploitation). L'AB doit permettre une meilleure valorisation d'une production moindre ou différente, d'autant que dans ces cas, l'ensemble du ménage est impliqué sur l'exploitation et qu'il n'y a pas d'autres sources de revenu. Directement ou progressivement, l'ensemble de l'exploitation est passé en AB. La certification s'accompagne d'un changement de mode de production, elle modifie profondément la vision de l'activité et s'accompagne, au moins pour les deux cas qui poursuivront dans l'AB, de changements dans les pratiques familiales dans le sens du bio.

La certification $\mathrm{AB}$ enclenche un cheminement dans l'AB de l'exploitation, mais aussi dans le bio des exploitants, voire de leur famille.

\section{Encadré 2. Cas de G}

Deux associés produisent des agneaux (300 brebis allaitantes) avec 40 ha de prairies, 2 ha de céréales et 350 ha de parcours, ainsi que du lavandin (2 ha). Un des associés décide d'arrêter en 1996 et vend la moitié du troupeau. Pour faire face à cette diminution brutale et inattendue du collectif de travail, l'épouse de l'exploitant restant, qui jusquelà occupait un emploi extérieur, décide de venir travailler sur l'exploitation. Ne bénéficiant plus d'un salaire extérieur, l'exploitation doit faire vivre la famille. Des choix sont faits : augmenter la taille du troupeau de 150 à 250 brebis, remplacer le lavandin par la lavande, mieux valorisée, tout en accroissant la surface de production par défrichage de terres incultes. Un collègue proche passé en $\mathrm{AB}$ et les prix proposés par une société intéressée par de la lavande bio (le double du prix du conventionnel) incitent l'exploitant à implanter cette production en AB en 1996. L'exploitant et son épouse étant satisfaits, ils commencent alors à faire des essais sur les autres productions (compost à la place des engrais, homéopathie pour le troupeau...) et font également évoluer leurs pratiques familiales en ce sens. Cela correspond à la façon dont ils ont envie de vivre et travailler. Ils passent ensuite l'ensemble des surfaces en $\mathrm{AB}$ en 1999, et dans un troisième temps le troupeau en 2000. Ils souhaitent rester en $\mathrm{AB}$, même si les sécheresses et le dépérissement de la lavande ont été difficiles économiquement, obligeant madame à reprendre un emploi extérieur. Face à une valorisation insuffisante des agneaux par la coopérative, ils passent à la vente directe. 


\section{Encadré 3. Cas de $\mathrm{H}$}

Cet exploitant s'installe en 1981, son projet est de récupérer les brebis de son père et de passer de 100 à 300 bêtes. C'est la crise ovine, alors son père propose de lui céder 14 ha pour la production de semences. En 1996, il récupère la totalité de l'exploitation et d'autres terres (70 ha et 100 brebis). Après avoir perdu les contrats pour les semences (car il n'était pas dans un îlot - zone reconnue de production avec un isolement pour éviter la contamination par d'autres cultures), il passe aux tomates d'industrie, et à la suite d'une année de surproduction, aux plants de tomates. Au moment des CTE bio, un conseiller lui dit qu'à part pour sa production de plants de tomates, il aurait peu à modifier ses pratiques pour passer en $A B$, et que cela représente une « sacrée manne financière ». Son épouse qui a un emploi extérieur, sensible à l'AB, le pousse aussi en ce sens, d'autant qu'il commence à avoir des problèmes pulmonaires

\section{- L'opportunité (2 cas)}

Dans ces cas, la décision du passage à l'AB intervient à une période où l'exploitant n'a pas de problème particulier, que ce soit au plan individuel, familial ou au niveau de l'exploitation. Jusqu'alors il n'avait pas envisagé l'AB comme une option pour son exploitation. C'est avant tout un argument économique qui amène à la certification (manne financière des CTE bio comme pour $\mathrm{H}$, encadré 3 ; demande de la filière), ainsi que l'influence de relations décisives. Ces cas correspondent à des logiques de développement de l'exploitation sur un mode entrepreneurial (par calcul économique et opportunité). La certification concerne la totalité de l'exploitation dans un cas, et seulement la production jugée la plus avantageuse et la moins difficile à pratiquer dans l'autre. Elle s'accompagne d'un changement de pratiques, alors que les individus eux-mêmes restent dans une logique entrepreneuriale.

La certification enclenche une trajectoire dans l'AB de l'exploitation et des qu'elle impute à l'utilisation de produits phytos. Mais pour lui l'AB est associée « aux cheveux longs ». Le conseiller lui démontre que c'est juste une autre façon de travailler, plus tournée vers le futur. En 2001, un collègue qui travaillait comme lui, passé en $\mathrm{AB}$, achève de le convaincre d'arrêter les tomates et de souscrire un CTE bio sur la totalité de l'exploitation. Cette aide financière lui permet de construire un bâtiment et de réaliser enfin son projet de passer à 300 brebis. En 2006, à la fin du CTE, la situation économique est difficile alors il recommence à faire des semences potagères, en $A B$ cette fois. Sans son épouse (pour le pousser et surtout s'occuper des papiers) et la manne financière, il ne serait pas passé en $A B$, car cela représente pour lui une surcharge administrative, et comme il le dit : «Si demain je divorce ou que le bio se casse la gueule, c'est pas dit que je retournerai pas en conventionnel.»

pratiques, sans changement de logique des individus et notamment sans enclencher un cheminement vers les principes du bio.

- La résolution de tensions au niveau individuel (8 cas)

Dans ces cas - les plus nombreux dans notre échantillon -, les exploitants sont pris dans des tensions, liées à des situations qu'ils ressentent comme non satisfaisantes et qu'ils souhaitent faire évoluer. Ces tensions sont économiques ( 2 cas), identitaires (4 cas) ou les deux ( 2 cas). C'est le passage à l'AB qui permet de les dénouer, à la suite d'un événement biographique (ex. : une terre « devenue orange » après application d'un désherbant), une opportunité (ex. : demande de la coopérative de commercialisation comme dans le cas de L, encadré 4), ou quand les conditions sont réunies (informations, réseaux, observation de tests concluants chez d'autres agriculteurs, négociations entre associés dont les avis divergent quant au passage à l'AB et test). On notera alors que les opportunités ne se restreignent pas à une vision 


\section{Encadré 4. Cas de L}

Âgé de 40 ans, cet agriculteur cultive 35 ha dont 25 ha de vergers. Son frère et son épouse, salariés de l'exploitation, sont favorables à l'AB. Ils consomment des produits issus de l'AB. Il a conscience du paradoxe : acheter en magasin bio, mais faire des traitements sur ses terres ! Même s'il prend beaucoup de précautions, il est soucieux de l'image qu'il donne (« je ne suis pas fier quand je suis sur mon tracteur»). De plus, il soupçonne que la maladie neurologique qui affecte son père, qu'il a vu traiter toute sa vie sans aucune précaution, soit en rapport avec l'usage des produits phytos. Alors quand la coopérative où il commercialise ses fruits lui demande s'il serait intéressé pour convertir ses 4 ha de kiwis en $\mathrm{AB}$, il saisit l'opportunité et se lance en 2009, parce que c'est une production facile à conduire en $\mathrm{AB}$ et que, coopérateurs de père en fils, il n'envisageait pas de commercialiser lui-même ses productions AB. Cela lui permet de se positionner dans une forme d'agriculture qu'il estime porteuse et d'avenir, alors que d'autres agriculteurs de son âge « continuent à traiter sans réfléchir et seront un jour montrés du doigt ». Dès qu'il y a eu la demande de la coopérative, il n'a pas hésité. Il envisage à l'avenir de convertir d'autres fruits (il raisonne en ce sens le choix des variétés qu'il replante), mais attend que la demande vienne de la coopérative, car il ne souhaite pas prendre en charge le conditionnement et la commercialisation. opportuniste de l'AB, mais c'est aussi ce qui a pu aider des exploitants à franchir le pas qu'ils n'arrivaient pas à franchir seuls, et enclencher un cheminement plus long dans l'AB voire dans le bio. Les exploitants ont exprimé la nécessité qu'ils ont ressentie de redonner du sens à leur métier, de (re) trouver une cohérence entre leur mode de vie familiale et leur exploitation, entre leur vision du métier et leurs pratiques, entre leur image de soi et celle perçue par les autres. La famille peut aider à impulser ce changement ou bien elle évolue en même temps que l'exploitation. Les changements de pratiques peuvent être limités, la certification n'être que partielle (pour tester et prendre de l'assurance), par contre la bifurcation amorcée s'affirme au niveau des individus quant à la vision de leur métier et de la façon de conduire leur activité.

La certification vient affirmer un cheminement déjà enclenché chez les individus, au niveau cette fois de l'exploitation et des pratiques, et conforte ou enclenche également celui des familles.

\section{Nature de la première certification}

Sur les 18 exploitations enquêtées, neuf ont réalisé directement une certification totale et dans neuf cas la première certification n'a été que partielle. Les certifications totales comme partielles se retrouvent dans les quatre types de circonstances. Il ne semble donc pas y avoir de lien simple entre les circonstances de la première certification $\mathrm{AB}$ et sa nature (totale/partielle). Dans les cas d'officialisation, quatre exploitants sur cinq ont fait une certification directe et totale. Ces éleveurs avaient l'ensemble de leurs pratiques qui répondaient déjà au cahier des charges de l'AB. Mais le cinquième cas - un arboriculteur - n'a fait pour commencer qu'une certification partielle, en raison de difficultés techniques non résolues sur certaines espèces fruitières, malgré son cheminement ancien dans le bio.

Dans les cas d'opportunité, on peut comprendre que la certification soit partielle si l'opportunité ne concerne qu'une production. Pour les autres circonstances, le lien avec le type de certification est moins évident.

La nature de la première certification semble davantage reliée au système de production et à la combinaison des activités. Dans les cas d'arboriculture ou dans des cas de combinaison d'activités multiples (élevage, culture, activités para-agricoles), 
la première certification a pu être partielle alors que dans les cas d'élevages herbivores seuls, même en présence d'une activité de transformation et vente, les certifications ont été totales. Dans les cas de vente directe ou d'accueil, la certification a fini par concerner la totalité de l'exploitation et on peut émettre l'hypothèse d'une importance de l'image renvoyée aux clients.

\section{Suite du processus : les certifications partielles dans les cheminements}

Parmi les neuf cas où la première certification était partielle, trois ont certifié progressivement leur exploitation jusqu'à sa totalité, trois ont certifié progressivement, mais partiellement leur exploitation. Trois n'ont fait pour l'instant que cette première certification partielle, il s'agit d'exploitations arboricoles. Deux parmi elles (L et $\mathrm{N})$ étaient en cours de conversion au moment de l'enquête. Il n'est pas possible de savoir, à ce stade, si d'autres certifications partielles seront réalisées à l'avenir, mais aux dires des exploitants nous pouvons émettre des hypothèses quant à la suite du processus. Pour l'ensemble de ces neuf cas, nous avons cherché à comprendre ce que signifiait la succession de certifications partielles dans les cas où cela s'est produit, et pourquoi certains, au moment de l'enquête, n'ont qu'une partie de leur exploitation certifiée AB.

\section{Certifications partielles successives Quelles significations?}

Les successions de certifications partielles, au regard de leur place dans les cheminements apparaissent au moment de l'étude comme des transitions, et ont été des tests avant d'aller plus loin. Ces transitions semblent rendre compte, dans les cas rencontrés, de trois types de cheminements qui relèvent (i) de la précaution, (ii) de la résolution de difficultés ou d'attentes d'opportunités, ou (iii) de la négociation. Lorsque la certification est partielle par précaution (cas G, J et E), les exploitants testent l'AB sur une portion de leur exploitation et expérimentent de nouveaux modes de conduite. La certification d'une nouvelle portion de l'exploitation se fait quand la précédente a donné satisfaction techniquement et économiquement.

Dans d'autres cas, ce sont des difficultés techniques qui freinent le développement de l'AB dans l'exploitation. Une nouvelle certification se produit quand les difficultés sont levées, par l'exploitant lui-même ou par les organismes de recherche et de développement. C'est le cas de l'arboriculteur $\mathrm{O}$ chez qui l'AB n'a été étendue à l'ensemble de l'exploitation que 12 ans après la première certification. Certains exploitants attendent aussi des opportunités, notamment commerciales, pour ne pas avoir à s'occuper eux-mêmes de la vente directe de leurs productions. Nous faisons l'hypothèse que le cas de L (encadré 4) s'inscrit dans un tel cheminement, car cet arboriculteur a exprimé le souhait de poursuivre le processus de certification $A B$ dès que sa coopérative lui en aurait fait la demande. Il prévoit d'ailleurs d'anticiper en choisissant des variétés adaptées à l'AB lors du renouvellement de ses vergers.

La négociation (cas I) quant à elle rend compte d'un cheminement différencié entre plusieurs individus sur une même exploitation (de forme sociétaire), certains favorables à l' $\mathrm{AB}$ et d'autres réticents. Il s'agit, pour les individus considérant l' $\mathrm{AB}$ comme une alternative intéressante, de se faire entendre. L'AB est alors testée sur une partie de l'exploitation, et la réussite de cet essai devient un argument dans le processus de négociation. Si les autres, souvent des associés, finissent par y voir un intérêt, bien souvent économique, une extension à d'autres surfaces ou productions est envisageable, et peut être facilitée si une aide financière la soutient. Mais les associés peuvent au contraire camper sur leurs positions et tolérer qu'une partie de l'exploitation soit en $\mathrm{AB}$ sans pour autant 
enclencher eux-mêmes un cheminement dans le bio. Nous formulons l'hypothèse que le cas $\mathrm{N}$ (en cours de conversion) va s'inscrire dans ce type de cheminement puisque l'exploitant a exprimé la nécessité que l'expérience en $\mathrm{AB}$ soit concluante sur les plans technique et commercial, pour convaincre son associé d'étendre la pratique de l'AB à d'autres cultures.

\section{Pourquoi des exploitants s'en tiennent} à une exploitation partiellement certifiée

Si nous faisons abstraction des deux cas en cours de conversion avec une certification partielle, quatre exploitants ont certifié tout ce qu'ils estimaient pouvoir certifier au moment de l'enquête. Pourquoi ne vont-ils pas plus loin et ne certifient-ils pas la totalité de leurs exploitations ?

Il s'agit dans les cas rencontrés soit de la persistance de difficultés et d'incertitudes quant à la certification du reste de l'exploitation générant une situation d'attente, soit d'une forme de rationalisation. Les difficultés et incertitudes énoncées peuvent concerner l'exploitation, qu'elles soient d'ordre technico-économique (ex. : changements profonds à mettre en place sur des ateliers qui fonctionnent bien tels quels : incertitude sur la maîtrise des maladies et adventices et risques à ne pas traiter), commercial (ex. : risque de perte de calibre des fruits par rapport à la demande de clients), ou liées aux contrôles. Les difficultés, lorsqu'elles relèvent des individus, sont d'ordre relationnel (on a déjà vu les freins que pouvait constituer le positionnement différent des associés par rapport à l' $\mathrm{AB}$ ) ou concernent le travail. Cela conduit les exploitants à prolonger la situation de certification partielle dans l'attente de circonstances plus favorables.

Mais cette stabilisation en certification partielle peut aussi témoigner d'une logique de rationalisation économique ou organisationnelle, rencontrée par exemple dans une exploitation de polyculture-élevage (cas I), avec une dissociation des raisonnements concernant les troupeaux et les surfaces. Là où, avant, il y avait autoconsommation par le troupeau des céréales produites sur l'exploitation, il y a maintenant production de céréales $\mathrm{AB}$ pour l'alimentation humaine (bien valorisées) et achat d'aliments conventionnels (moins chers) pour un troupeau non certifié AB. Les exploitants peuvent désormais jouer sur l'achat, la vente, la consommation de céréales $\mathrm{AB}$ et conventionnelles selon la fluctuation des prix, alors qu'avant il leur fallait valoriser correctement leurs animaux, seul produit fini. C'est le cas également de l'exploitant $\mathrm{P}$, pour lequel la certification correspond à une opportunité commerciale (une demande de kiwis bio pour l'exportation), et même s'il est sensibilisé aux problématiques environnementales, la certification $A B$ semble correspondre au choix d'une option parmi d'autres pour améliorer la rentabilité de l'exploitation. Ces exploitants sont davantage dans un raisonnement d'optimisation économique que de recherche d'autonomie. Certains jouent également sur le choix des productions, se donnant la possibilité d'en changer selon les valorisations escomptées, ou de ne certifier que les surfaces intéressantes (par exemple l'exploitant J a laissé les surfaces non accessibles à la moissonneuse en conventionnel, car elles serviront au pâturage du troupeau qui n'est pas en $\mathrm{AB}$, tout en déplorant que les fourrages issus de terres $\mathrm{AB}$ ne soient pas bien valorisés, au sens économique, par ce même troupeau !). Cette logique de rationalisation est plus ou moins poussée selon le cheminement des individus. Pour certains il s'agit avant tout de jouer sur plusieurs tableaux, celui du conventionnel et celui de l'AB, de faire ce qui leur paraît le mieux pour les troupeaux, les terres, comme pour les individus. Compte tenu de leurs connaissances et de la situation à laquelle ils ont à faire face, le recours raisonné à des intrants chimiques dans une approche globale du système ne leur paraît pas forcément dommageable. 
RECHERCHES

Françoise ALAVOINE-MORNAS, Sophie MADELRIEUX

\section{Comment une approche par les processus renouvelle l'appréhension des passages à l'AB}

Cette approche longitudinale s'appuie sur la reconstruction par les interviewés de leur cheminement (Lamine et Bellon, 2009), le temps et les expériences traversées faisant évoluer le regard que les individus portent sur leur histoire. Ce qui nous a intéressées, plus que la seule matérialité des faits, est bien la façon dont les exploitants eux-mêmes redéfinissent leur histoire et celle de leurs activités, explicitant dans quels mouvements ils se situent et comment ils redéfinissent leur rôle (Lemery, 2011). Cette approche reste exploratoire et même si des tendances générales se dégagent, celles-ci devront être confrontées à d'autres études de cas pour mettre à l'épreuve ces premiers résultats. Toutefois il nous semble qu'elle permet d'accroître l'intelligibilité des passages à l'agriculture biologique, en donnant à voir une diversité de circonstances conduisant à une certification $A B$, de chemins parcourus par les individus, familles et exploitations, dépassant ainsi les approches par les motivations ou par les conséquences, et permettant de contribuer au débat sur les formes d'exercice de l'agriculture biologique.

\section{Diversité des processus}

Notre analyse a mis en évidence que les circonstances et la nature de la première certification $\mathrm{AB}$, la suite du processus, les cheminements des individus, des familles et des exploitations, sont autant d'éléments allant dans le sens de notre hypothèse selon laquelle l'engagement dans l'AB et le bio diffère en fonction du processus de passage à l' $\mathrm{AB}$, ce que nous abordons dans la suite. Cela nous a également permis de nuancer certaines affirmations de la littérature sur l'engagement dans l'agriculture biologique dans les cas de certifications récentes, et d'appréhender le rôle des familles et des reconstructions identitaires dans l'ancrage et la pérennisation de cet engagement.

\section{Au-delà des motivations}

Les circonstances du passage à l'AB

Dans les cas rencontrés, la certification $\mathrm{AB}$ n'apparaît finalement guère motivée, au sens de projetée, préparée, anticipée. Certes, dans les cas de tensions, un intérêt personnel ou familial pour le bio est entré peu à peu en tension avec les pratiques sur l'exploitation, et a pu conduire ainsi à une certaine motivation pour le passage à l'AB. Mais nous avons aussi montré ce que peuvent être des imprévus ou des opportunités, qui enclenchent une certification de tout ou partie de l'exploitation, alors que l'AB n'était pas envisagée au préalable comme une option de développement. La certification est également l'option choisie par les exploitants dans les cas d'officialisation où il s'agit de répondre à une demande alors que les pratiques répondent déjà au cahier des charges $\mathrm{AB}$, sans que les exploitants aient cherché jusque-là à entrer dans la démarche de labellisation.

\section{Cheminements des individus,} des familles, des exploitations

Une telle approche par les processus permet également de saisir les chemins parcourus et de repérer si, au niveau de l'exploitation, il y a juste une adoption basique des règles de l' $\mathrm{AB}$ ou une reconception du système (Sylvander et al., 2006), et si, pour les individus, il y a continuité ou au contraire rupture et mise en place d'une nouvelle façon de voir son activité (Van Dam et al., 2009). Elle accorde de l'importance au temps et aux évolutions (Padel, 2001) et montre que, dans certains cas rencontrés, si, au départ, c'est seulement l'exploitation ou une partie de celle-ci qui est en $A B$, les individus finissent par enclencher un cheminement personnel vers le bio (cf. encadré 2), par contact avec l'AB. 


\section{Les certifications partielles}

En accordant de l'importance à la signification de la certification $\mathrm{AB}$ dans les cheminements, nous comprenons mieux également les certifications partielles. Audelà de tests et d'expérimentations, en vue de prendre son temps et de faire sa propre expérience, tel que déjà noté par Padel (2001), les certifications partielles apparaissent également comme le résultat de négociations entre associés d'une même exploitation. Et lorsqu'elles se stabilisent dans la durée, elles témoignent de difficultés et d'incertitudes non encore résolues, d'attentes ou d'une logique de rationalisation sur les plans économiques et du travail.

\section{Les certifications récentes}

Dans de nombreux travaux, notamment sur la conventionalisation de l' $\mathrm{AB}$, on peut lire que les exploitants qui se sont convertis depuis la fin des années 1990 y auraient été incités par des motifs principalement économiques (Padel, 2001 ; Rigby et Caceres, 2001). Or, dans les exploitations récemment certifiées de notre enquête, nous avons rencontré des individus qui ont adopté de longue date un mode de vie et de travail bio. Ceci suggère que certains exploitants bio se mettent dans les rangs de la certification $\mathrm{AB}$, car il devient difficile pour eux d'exercer leur activité, de parler de leurs pratiques en disant qu'elles sont bio si le logo vert $A B$ n'est pas présent, alors même qu'ils sont peu satisfaits des évolutions de l'AB et de son cahier des charges.

\section{Rôle de la famille \\ et des reconstructions identitaires}

Nous avons formulé l'hypothèse que, selon le processus de passage, l'engagement dans l'AB et le bio n'est pas le même. Il ressort des cas étudiés que l'engagement dans l'AB semble davantage s'ancrer, et pouvoir se pérenniser, si la famille s'engage également dans un cheminement vers les principes du bio, ou si les circonstances à l'origine du passage à l'AB sont liées à des tensions identitaires que la certification $\mathrm{AB}$ contribue à résoudre.

En effet, la famille joue des rôles différents dans les processus de passage à la certification AB. Dans certains cas, elle apparaît peu concernée par ce qui se passe sur l'exploitation, mais dans la majorité des exploitations enquêtées, son poids semble important dans le passage à l'AB. La famille peut être à l'origine de ce passage, dans les cas d'imprévus, ou jouer un rôle décisif d'incitation. Elle peut aussi soutenir et encourager le processus (prise en charge des aspects administratifs par l'épouse). L'adoption de pratiques bio par la famille (alimentation, santé, consommation d'énergie) peut précéder la certification $\mathrm{AB}$ de l'exploitation, et engendrer des tensions identitaires chez les exploitants. La famille peut, dans d'autres cas, être influencée par le passage à l'AB, suivre le processus sur l'exploitation, se questionner sur ses propres pratiques et enclencher un cheminement vers le bio. C'est dans ces cas, où la famille enclenche un cheminement vers le bio ou a déjà des pratiques bio, qu'un cheminement de l'exploitation dans l'AB, voire dans le bio, semble se renforcer. Ainsi, la transformation des exploitations reste marquée par la famille même lorsque celle-ci ne travaille plus sur l'exploitation (Madelrieux et al., 2011). La résolution de tensions sur le plan individuel paraît être aussi une voie d'engagement dans l'AB, constituant pour certains un moyen de sortir de la crise du métier et d'enclencher un processus de reconstruction identitaire (Van Dam, 2005), où $A B$ et bio viennent redonner du sens au métier, ainsi qu'une cohérence entre pratiques professionnelles et éthique personnelle, laissant penser que l'engagement sera plus pérenne.

\section{Formes d'exercice de l'agriculture biologique}

Sur le plan méthodologique, cette approche par les processus présente plusieurs intérêts, qui justifient son choix et son utilisation dans ce travail. Elle prend en compte 
les liens et les influences réciproques entre individus, familles, exploitations et environnement socio-économique. Elle distingue différentes pratiques et différents engagements dans l'agriculture biologique : le bio (au sens des principes fondateurs), l'AB (au sens de la réglementation européenne), le conventionnel (au sens de non $\mathrm{AB}$ et « non bio »), et leurs combinaisons (certifications partielles/totales). Elle décortique les transformations (des individus, des familles, des exploitations) en accordant de l'importance aux temporalités en deçà et au-delà de la période de conversion officielle. Et par là même, elle aborde frontalement la complexité des passages à l'agriculture biologique et de ses formes d'exercice, en contribuant à déconstruire les dualismes simplificateurs du genre $\mathrm{AB}$ vs conventionnel ou éthique $v s$ opportunisme. Elle confirme également l'intérêt de conduire un raisonnement en types de processus plutôt qu'en types de producteurs, comme c'est le plus souvent le cas dans la littérature (Lamine et Bellon, 2009). En effet, les typologies de producteurs biologiques, bien souvent basées sur les motivations à pratiquer l'AB, masquent, d'une part, la diversité des circonstances par lesquelles les exploitants en viennent à l'AB (Darnhofer et al., 2005) et, d'autre part, les chemins parcourus.

Cette approche par les processus propose non pas d'évaluer si la façon dont est mise en œuvre l'agriculture biologique est durable, mais d'identifier les directions prises (Rigby et Caceres, 2001), les chemins parcourus au niveau des individus et des exploitations. Cela constitue une autre façon d'évaluer ou de promouvoir des formes d'exercice de l'agriculture biologique, qui présente les avantages de reconnaître la diversité des modes d'appropriation des exploitants, et d'élargir le champ d'action en donnant à voir les formes intermédiaires et en transition. La littérature a accordé peu d'importance à la diversité de ces formes d'exercice de l'agriculture biologique, au profit de comparaisons de situations stabilisées : entre $\mathrm{AB}$ et conventionnel, entre avant et après la certification (Lamine et Bellon, 2009). Pourtant, entre les extrêmes - d'un côté, des individus bio avec des exploitations bio et totalement en $\mathrm{AB}$ et, de l'autre, des individus non motivés par les principes du bio avec des exploitations partiellement en $\mathrm{AB}$ dans une logique de rationalisation -, existe tout un gradient de situations intermédiaires. Il nous semble porteur, à l'instar de Darnhofer et al. (2010), d'analyser ces formes intermédiaires susceptibles de cheminer dans l'AB et le bio, et d'ouvrir le champ des possibles, plutôt que de se focaliser sur des situations extrêmes supposées stables. C'est alors reconnaître les réalités multiples des exploitants en déplaçant l'attention sur le « comment ». Comment les agriculteurs s'approprient l'AB ? Comment ils transforment et reconstruisent sa signification (Darnhofer et al., 2005) ? Comment ils inventent des « arts de faire » et se réapproprient les usages à leur façon (de Certeau, 1990) ? Pour certains des exploitants rencontrés, il s'agit avec l'AB de cheminer pour redonner du sens à leur métier et notamment retrouver l'autonomie et le local («bio et local, c'est l'idéal» comme clame l'un d'eux), pour d'autres, il s'agit de sortir de la routine, de se donner un nouveau défi, d'arriver à faire aussi bien en $\mathrm{AB}$ qu'en conventionnel en termes de productivité, de se faire une place dans un collectif, pour d'autres encore l'AB est une étape sur le chemin accidenté de la vie.

Cette importance accordée aux formes intermédiaires, en transition, aux chemins parcourus par les individus et les exploitations, peut-elle contribuer à renouveler les discussions sur les formes d'exercice de l'agriculture biologique ? Elle suggère, dans une perspective de développement de l'AB, de promouvoir une agriculture biologique non pas opposée à l'agriculture conventionnelle (ce qui aiderait à minimiser les réactions négatives chez les exploitants conventionnels ; Padel, 2001), mais constituée d'une diversité de formes possibles 
d'exercice de l'activité. Elle montre les continuités, les passages entre agriculture conventionnelle et agriculture biologique, les enracinements dans la tradition (Barrès et al., 1985). Elle propose enfin de porter attention à la façon dont les exploitants réinventent leurs modes de production, font évoluer leurs relations aux autres, expérimentent, observent..., tout ce qui ressort chez les interviewés comme stimulant, comme redonnant du sens à leur activité.

\section{Conclusion et perspectives}

Pour clarifier ce que certains nomment « conversion à l'agriculture biologique », nous avons écouté des histoires de vie, nous avons saisi des tensions qui s'actualisent, s'intensifient, se résolvent, se déplacent; des difficultés et des incertitudes, mais aussi des désirs et des projets ; des rythmes différents, des prises de conscience plus ou moins rapides ; des interactions entre des individus, leur famille et ses activités, leur environnement. Nous avons cherché à comprendre les processus à l'œuvre, en choisissant d'accorder plus d'importance aux chemins parcourus qu'aux états à un moment donné. Nous avons tenu à nous affranchir des dualismes très présents dans la littérature et qui ne prennent pas en compte les situations intermédiaires. Nous avons voulu aussi attirer l'attention sur le fait que les opportunités de passage à l'AB ne relèvent pas que d'une vision opportuniste, mais sont quelquefois décisives pour franchir un pas qu' on n' arrive pas à franchir seul. Ces éléments, qui permettent une compréhension affinée des passages à l'AB, pourraient être utilisés pour une adaptation des politiques publiques et des actions de soutien à l'agriculture biologique : à ce titre, les acteurs publics et ceux du développement agricole pourraient utilement s'en saisir. Toutefois, la dynamique de l'AB dépend non seulement des entrées, mais également des sorties de l'AB. Parmi les exploitants rencontrés, l'un a finalement choisi de quitter l' $\mathrm{AB}$, un autre a été contraint par l'organisme certificateur de décertifier une partie de son exploitation. Cela nous interroge sur les facteurs influençant le maintien en $A B$, et les raisons de sortie de l'AB. Ainsi, l'un des prolongements que nous avons donnés à ce travail est d'étudier l'ampleur du phénomène de sortie de l'AB et les processus à l'œuvre et, au travers de l'analyse du maintien dans la certification, d'approfondir l'hypothèse que selon le processus de passage, l'engagement dans l'AB n'est pas le même.

\section{RÉFÉRENCES BIBLIOGRAPHIQUES}

Agence Bio. (2012). L'agriculture biologique française : les chiffres clés. Édition 2012 (Chiffres 2011), 260 p.

Barres D., Bonny S., Le Pape Y., Remy J. (1985). Une éthique de la pratique agricole. Agriculteurs biologiques du Nord-Drôme. Paris, INRA Économie et Sociologie rurale, 109 p.

Bertaux D. (1997). Les récits de vie. Perspective ethnosociologique. Paris, Nathan Université, $128 \mathrm{p}$.

Darnhofer I., Lindenthal T., BartelKratochvil R., Zollitsch W. (2010). Conventionalisation of organic farming practices: from structural criteria towards an assessment based on organic principles. Review Agronomy for Sustainable Development, $\mathrm{n}^{\circ} 30$, p. 67-81.

Darnhofer I., Schneeberger W., Freyer B. (2005). Converting or not converting to organic farming in Austria: farmer types and their rationale. Agronomy Human Values, $\mathrm{n}^{\circ} 22$, p. 39-52.

De Certeau M. (1990). L'invention du quotidien. Arts de faire. Paris, Gallimard, 350 p.

Evans N. (2009). Adjustment strategies revisited: agricultural change in the Welsh 
Marches. Journal of Rural Studies, $\mathrm{n}^{\circ} 25$, face à l'innovation ", Paris, L'Harmattan, p. $217-230$ p. 243-254.

Fairweather J. (1999). Understanding how farmers choose between organic and conventional production: results from New Zealand and policy implications. Agronomy Human Values, $\mathrm{n}^{\circ} 16$, p. 51-63.

Guthman J. (2000). An agro-ecological assessment of grower practices in California. Agronomy Human Values, $\mathrm{n}^{\circ} 17$, p. 257-266.

Guthman J. (2004). The trouble with organic lite in California. A Rejoinder to the conventionalisation debate. Sociologia ruralis, $\mathrm{n}^{\circ}$ 44, p. 301-316.

Harris C. K., Powers S. E., Buttel F. H.. (1979). Myth and Reality in Organic Farming: A Profile of Conventional and Organic Farmers in Michigan. Annual Meeting of the Rural Sociology Society, Burlington, Vermont, August.

IFOAM. (2005). Principles of organic agriculture. Bonn, IFOAM (International Federation of Organic Agriculture Movements, 4 p., http://www.ifoam.org/

Koesling M., Loes A. K., Flaten O., Kristensen L. H., Hansen M. W. (2012). Farmers' reasons for deregisterinf from organic farming. Organic Agriculture, June 2012, vol. 2, issue 2, p. 103-116.

Lamine C., Bellon S. (2009). Conversion to organic farming: a multidimensional research object at the crossroads of agricultural and social sciences. Review Agronomy for Sustainable Development, $\mathrm{n}^{\circ}$ 29, p. 97-112.

Lamine C., Meynard J.-M., Perrot N., Bellon S. (2009). Analyse des formes de transition vers des agricultures plus écologiques : les cas de l'agriculture biologique et de la production intégrée. Innovations agronomiques, $\mathrm{n}^{\circ} 4$, p. 483-493.

Lamine C. (2011). Transition pathways towards a robust ecologization of agriculture and the need for system redesign. Cases from organic farming and IPM. Journal of Rural Studies, ${ }^{\circ} 27$, p. 209-219.

Lemery B. (2011). Les agriculteurs : une profession en travail. In Béguin P., Dedieu B., Sabourin E. (dir.), «Le travail en agriculture : son organisation et ses valeurs

Le Pape Y., Remy J. (1988). Agriculture biologique : unité et diversité. In Jollivet M. (dir.), Pour une agriculture diversifiée (p. 134-140), Paris, L'Harmattan, coll. «Alternatives rurales ».

Madelrieux S., Nettier B., Dobremez L. (2011). Transformations des exploitations d'élevage au prisme des relations famille-travail. In Béguin P., Dedieu B., Sabourin E. (dir.), Le travail en agriculture: son organisation et ses valeurs face à l'innovation (p. 189-204), Paris, L'Harmattan.

Nelson E, Tovar L. G., Rindermann R. S., Cruz M. A. G. (2010). Participatory organic certification in Mexico: an alternative approach to maintaining the integrity of the organic label. Agronomy Human Values, $\mathrm{n}^{\circ} 27$, p. 227-237, doi : 10.1007/s10460-009-9205-x.

Padel S., Roecklingsberg H., Verhoog H., Alroe H., De Wit J., Kjeldsen C., Schmid O. (2007). Balancing and integrating basic values in the development of organic regulations and standards: Proposal for a procedure using case studies of conflicting areas. University of Wales, Aberystwyth, available on-line at: http://orgprints.org/10940/.

Padel S. (2001). Conversion to organic farming: a typical example of a diffusion of an innovation? Sociologia ruralis, ${ }^{\circ} 41$, p. 40-61.

Pettigrew A. M. (1990). Longitudinal field research on change: theory and practice. Organization science, $\mathrm{n}^{\circ} 1$, p. 267-292.

Rigby D., Cáceres D. (2001). Organic farming and the sustainability of agricultural systems. Agricultural Systems, n ${ }^{\circ}$ 68, p. 21-40.

Sylvander B., Bellon S., Benoît M. (2006). Facing the organic reality: the diversity of development models and their consequences on research policies. Odense (DK), Joint Organic Congress Organic Farming and Eur. Rural Development, 4 p.

Van Dam D. (2005). Les agriculteurs bio, vocation ou intérêt? Namur, Presses universitaires de Namur, $201 \mathrm{p}$.

Van Dam D., Nizet J., Dejardin M., Streith M. (2009). Les agriculteurs biologiques. Ruptures et innovations. Dijon, Éducagri Éditions, $141 \mathrm{p}$. 